\title{
New adiabatic calorimeter for realization the triple point of water in metallic-sealed cell at NIS-Egypt
}

\author{
Ahmed Ali El Matarawy^ and Mohamed Gamal Ahmed \\ National Institute for Standards, Tersa St., El Haram, Giza 12211, Egypt
}

Received: 30 November 2015 / Accepted: 9 March 2016

\begin{abstract}
The thermal metrology laboratory, at the National Institute for Standards (NIS-Egypt), has been working during the last three years on the development of metallic-sealed cells for the realization of the triple point of water (TPW), 273.16 K [H. Preston-Thomas, Metrologia 27, 3 (1990)]. A metallicsealed cell has been already developed and characterized in the past [A. El Matarawy, M.G. Ahmed, Int. J. Metrol. Qual. Eng. 5, 401 (2014), M.G. Ahmed, A. El Matarawy, H.M. Abo Dorra, J. Phys. Sci. Appl. 1 (2015)]. The classical large triple point of water cell and metallic-sealed cell showed a discrepancy of $0.7 \mathrm{mK}$ [A. El Matarawy, M.G. Ahmed, Int. J. Metrol. Qual. Eng. 5, 401 (2014)]. The main target of this work is to get the lowest possible uncertainty in calibrating Capsule Standard Platinum Resistance Thermometers (CSPRTs) under adiabatic conditions. The full system is composed of a metallic-sealed cell and an improved adiabatic calorimeter to increase as much as possible the thermal resistance between the cell and the inner adiabatic shield of the calorimeter. It gives also a reliable calculation of the heat capacity of the cell and the comparison copper block which accommodates several CSPRTs. With this new system the reproducibility of metallic-sealed cell was found to be $0.7 \mathrm{mK}$ as described below. reproducibility of metallic-sealed cells is found to be less than $0.7 \mathrm{mK}$.
\end{abstract}

Keywords: Adiabatic calorimeter, triple point of water (TPW), metallic cell, CSPRT, uncertainty

\section{Introduction}

The triple point of water is one of the defining fixed points of the International Temperature Scale of 1990 (ITS-90) [1], with an assigned temperature value of $T w=$ $273.16 \mathrm{~K}$. It is the most important defining thermometric fixed point used in the calibration of thermometers to the ITS-90, where it is the temperature to which the resistance-ratios given in SPRT calibrations are referred. It is also important for the redefinition of the kelvin based on Boltzmann constant. Since the Kelvin, as defined, is the fraction $1 / 273.16$ of the thermodynamic temperature of the triple point of water. Thus any change linked to the TPW will affect the definition.

From the previous work on metallic sealed cell $[2,3]$, the noted difference between classical large borosilicate glass and metallic cells was $0.7 \mathrm{mK}$. Thus it was decided to improve the calorimeter structure with a new one to increase as much as possible the thermal resistance between the cell and the inner shield to decrease the parasitic heat flux during the measurements. This was expected to improve the estimated uncertainty as well.

The discrepancy between the two cells is given by $\Delta T$ using the following equation:

$$
\Delta T=T_{T P}^{L C}-T_{T P}^{M C},
$$

${ }^{\star}$ Correspondence: ahmed.matarawey@gmail.com where, $T_{T P}^{L C}$ and $T_{T P}^{M C}$ are the triple point temperatures of the large cell and metallic cell respectively [3]. The $T_{T P}^{L C}$ was calculated from the plateau average value and in this case there is no way to control the melting fraction. On the other hand, the $T_{T P}^{M C}$ is calculated exactly at a fraction of $50 \%$ of solid ice melt [4].

\section{System design}

The full system is composed of two main parts; a compartement that includes the cell, copper comparison block and thermometer as one unit and a new designed calorimeter accomodates the compartement during several stages of thermalization.

\subsection{Metallic-sealed cell}

The compartment includes the TPW metallic sealed cell that is mounted in a good thermal contact with a cooper comparison block using good thermally conductor silver paste. This block contains several holes with different diameters to accommodate up to four thermometers at the same run as shown in Figure 1. The geometry of the cell has been chosen to be similar as those made for argon and oxygen triple point cells $[5,6]$. 

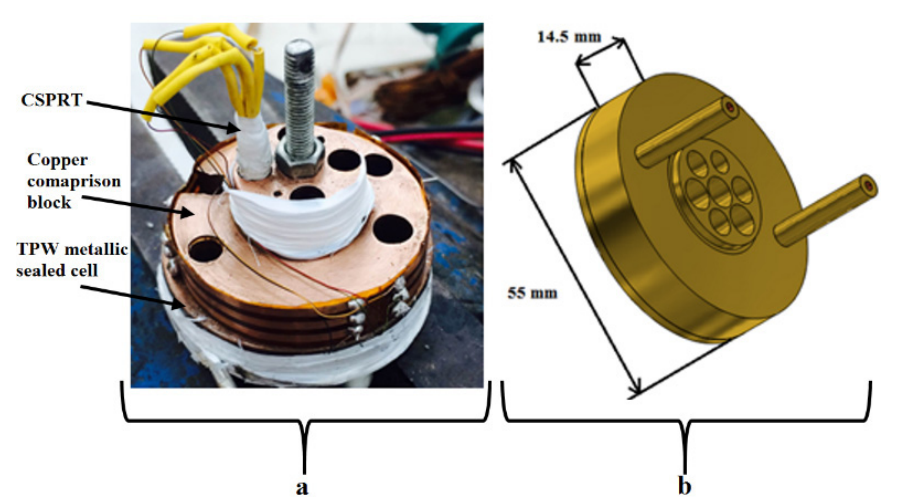

Fig. 1. (a) a compartment composed of TPW metallic sealed cell, copper comparison block and CSPRT. (b) Design of TPW metallic sealed cell.
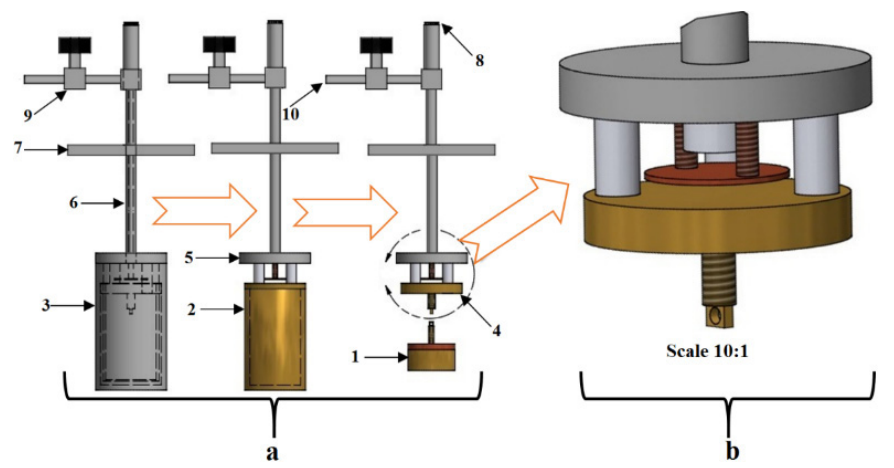

Fig. 2. New improved adiabatic calorimeter, (1) compartment of the TPW, CSPRT and copper comparison block, (2) copper regulation shield, (3) stainless-steel pressure vessel, (4) Brass flange, (5) stainless-steel flange, (6) main central tube, (7) rectangular stainless-steel flange, (8) wires feed through, (9) vacuum regulation valve, (10) to the vacuum pump.

\subsection{An improved adiabatic calorimeter}

The new calorimeter assembly is shown in Figure 2, it consists of a compartment (1) that is suspended by a silk wire. This wire is fixed to a brass disk (4). This disk is fitted to the Copper regulation shield (2) and thermally decoupled from the stainless-steel flange (5) by three Teflon rods. The vacuum can (3) is assembled with a flange (5). Evacuation was achieved through the central main tube (6) that is connected to a turbo-molecular pump.

\section{Thermal characterization of the cryostat}

Thermal energy can be transferred to the compartment (TPW, Copper block and the CSPRTs) by three ways:

\subsection{By convection}

Adiabatic condition was maintained at a pressure of $2.2 \times$ $10^{-6}$ mbar at the inner space between the compartment and the vacuum can. This ensure goodthermal resistance in the space between the cell and the shield.

\subsection{By conduction}

One important modification from the previous calorimeter to the new one is to replace stainless steel screws that links the brass disk with stainless steel flange of the vacuum can (3) with Teflon rods as shown in Figure 2b. Wires of the CSPRTs and the heaters were glued to the cell and passed through three main thermalization stages from outside:

1. Thermalization at the inner face of the stainless steel flange (Fig. 2a [6]) that contains a first drilled copper rod and the wires wound around it.

2. Thermalization at the inner face of the brass disk (Fig. 2a [5]) that contains a second copper rod.

3. A Copper thermal sinking sheet wound circularly around the cell (Fig. 1) to electrically connect and ensure the thermal dissipation of heat in the wires and the CSPRTs.

\subsection{By radiation}

New thermal screen made of copper was mounted on the front of the main central evacuation tube to absorb any parasitic heat flux coming from the pump or the ambient.

\section{Experimental arrangements}

At the start of the experiment, the CSPRT which was intended to be calibrated in the TPW cell was mounted into the new modified calorimeter. An alcohol bath "Fluke Model 7381 " with a stability of $0.006{ }^{\circ} \mathrm{C}$ was used for the realization of TPW. Measurements of CSPRT ("Tinsley code UUT") were obtained using an F18 ASL resistance bridge in conjunction with a $100 \Omega$ (calibrated value $100.000442 \Omega$ ) Tinsley standard resistor maintained in a thermostated oil bath at $23{ }^{\circ} \mathrm{C}$. The UUT has been calibrated according to the ITS-90 as shown in Figure 3. This thermometer was chosen after showing a good stability better than $0.1 \mathrm{mK}$ over several years at the TPW.

A multimeter (Fluke Model 8864A) was used to measure the resistance of the $\mathrm{Pt}-100$ which is placed at the top side of a brass disk on the copper thermal shield and a multimeter (Keithley Model 2000) was used to measure the resistance of Pt-1000 at the bottom side of that brass disk. A current source (Keithley Model 2400) was used to pass electric current to the heater $(75 \Omega)$ of the thermal shield when controlled through the PID software. Another current source (Keithley Model 224) was used to pass electric current to the heater $(46 \Omega)$ that wound around the compartment.

\section{Metrological procedures}

In order to realize the TPW under adiabatic conditions (intermittent heat pulse technique) the following procedure was used. 


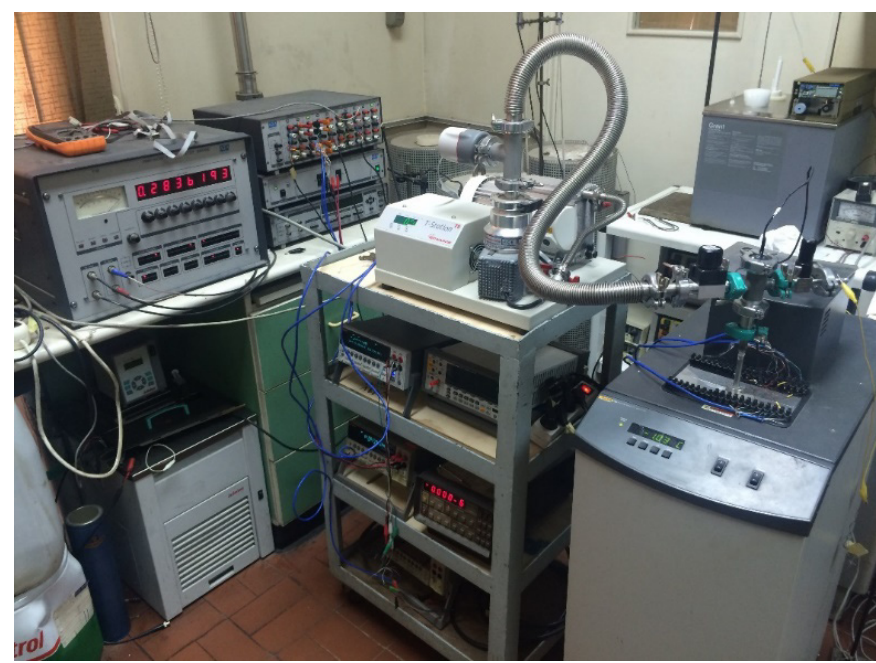

Fig. 3. Experiment setup of the new adiabatic calorimeter that realizes the TPW.

Firstly, the capsule thermometer was fixed in the compartment then both of them were inserted into the calorimeter. The calorimeter was controlled by a PID software working under labview environment. The metallicsealed cell was allowed to be completely solid after monitoring the supercool of the cell which happened at $-7{ }^{\circ} \mathrm{C}$, The copper shield temperature was raised up to some hundreds of millikelvins just below the triple point temperature and at this point helium exchange gas was pumped out. The temperature of the controlled shield was raised again to be few millikelvin above the plateau point, with different set points, but the temperature of the compartment is still below the triple point because the system has a thermal time constant of several hours. Therefore, the temperature of the triple point cell was raised quickly by feeding the heater wound around the cell until melting of the solid water inside the cells begins. Class A Pt-1000 controlled the temperature of the shield and the cell temperature was monitored by the UUT capsule thermometer. An intermittent heat pulsing was started to melt all quantity of water inside the cell fraction by fraction. Experimental data was corrected for thermometer self-heating effect using measurements at $1 \mathrm{~mA}$ and $\sqrt{2} \mathrm{~mA}$.

The current source starts automatically feeding series of pulses. The optimum conditiond after several trials were found to be by feeding current equivalent to $38.869 \mathrm{~mA}$ during $25 \mathrm{~min}$ and the relaxation time took $285 \mathrm{~min}$ per one pulse as shown in Figure 4 in upper part and zoomed in lower part.

Therefore, the thermal energy applied through one pulse is equivalent to $104.245 \mathrm{~J}$ and the total provided energy for full plateau realization is equivalent to 1563.678 J. Overheating after each heat pulse started from $+3.7 \mathrm{mK}$ at the first melting fraction up to $60 \mathrm{mK}$ at the end of the melting plateau as shown in Table 1. After the cell was fed with small amount of thermal energy, the temperature increased rapidly from $T_{\text {Plateau }}$ to $T_{\text {Pulse }}$ then the temperature was decayed exponentially again to the $T_{\text {Plateau values. The minor part of that thermal energy }}$
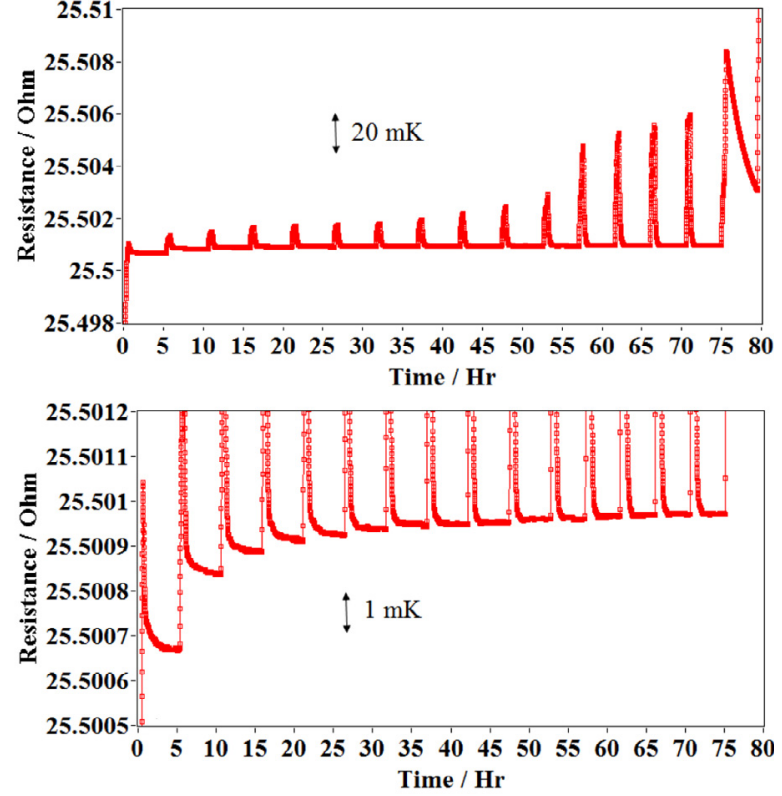

Fig. 4. TPW realization by intermittent heat pulse technique.

causes increasing in temperature of the sensing element of the thermometer and the major part was used to melt the solid ice inside the cell at the beginning of plateau, so an overheating shown as maximum temperature (maximum of the peak appeared after each pulse) gives a quantitative indication about the amount of the solid ice inside the cell. The correlation between the overheating and the melted fraction is a linear relationship as shown in Figure 5. Plateau duration was approximately $77 \mathrm{~h}$ and the triple point at $50 \% F$ is $25.50095 \Omega$ which is equivalent to $273.1601 \mathrm{~K}$ giving $0.1 \mathrm{mK}$ temperature difference as shown in Table 1.

The CSPRT resistance has been measured at each melted fraction $F$ after each heat pulse as shown in Figure 6 . Results from measurements at $F$ ranging from $20 \%$ up to $80 \%$ have been extrapolated from a first-order polynomial fit at the liquidus point as shown in Figure 7 for one run. The reproducibility of the metallic cell was deduced from the width of the different extrapolation fits for the different runs.

Table 1 gives the order of the energy pulses fed to the cell with respect to the average value of the plateau resistance, the maximum temperature after each heat pulse, the consuming time for the temperature to decay exponentially from $T_{\text {Pulse }}$ to $T_{\text {Plateau }}$, the temperature difference between overheating temperature $T_{\text {Pulse }}$ and $T_{\text {Plateau }}$ for each pulse and finally the temperature difference between $T_{\text {Plateau }}$ and $T_{90}$.

\section{Thermal study of the calorimeter}

In order to estimate the quality of the improved adiabatic calorimeter, a characterization study is carried out as in references $[4,7,8]$. There are three main thermal factors of the study describing the thermal behaviour 
Table 1. Summary of the data analysis of TPW realization.

\begin{tabular}{cccccc}
\hline $\begin{array}{c}\text { Pulse } \\
\text { order }\end{array}$ & $\begin{array}{c}\text { Plateau } \\
\text { value }(\Omega)\end{array}$ & $\begin{array}{c}\text { Overheating } \\
(\Omega)\end{array}$ & $\begin{array}{c}\text { Decay } \\
\text { time }(\min )\end{array}$ & $\begin{array}{c}T_{\text {pulse }}-T_{\text {plateau }} \\
(\mathrm{m} \mathrm{K})\end{array}$ & $\begin{array}{c}T_{\text {cell }}-T_{90} \\
(\mathrm{~m} \mathrm{~K})\end{array}$ \\
\hline 1 & 25.50067 & 25.50104 & 6.18 & 3.66 & -2.64 \\
2 & 25.50085 & 25.50134 & 6.49 & 4.77 & -0.86 \\
3 & 25.50089 & 25.50152 & 6.71 & 6.14 & -0.45 \\
4 & 25.50092 & 25.50167 & 6.96 & 7.38 & -0.19 \\
5 & 25.50093 & 25.50173 & 7.22 & 7.80 & -0.08 \\
6 & 25.50094 & 25.50175 & 7.13 & 7.90 & 0.01 \\
7 & 25.50095 & 25.50182 & 7.29 & 8.52 & 0.09 \\
8 & 25.50095 & 25.50196 & 7.44 & 9.90 & 0.10 \\
9 & 25.50095 & 25.50187 & 7.39 & 9.03 & 0.13 \\
10 & 25.50096 & 25.50246 & 7.65 & 14.70 & 0.20 \\
11 & 25.50096 & 25.50292 & 7.89 & 19.24 & 0.21 \\
12 & 25.50097 & 25.50480 & 8.09 & 37.64 & 0.27 \\
13 & 25.50097 & 25.50527 & 8.27 & 42.30 & 0.28 \\
14 & 25.50097 & 25.50614 & 8.69 & 50.84 & 0.29 \\
15 & 25.50097 & 25.50707 & 8.87 & 60.00 & 0.31 \\
\hline
\end{tabular}

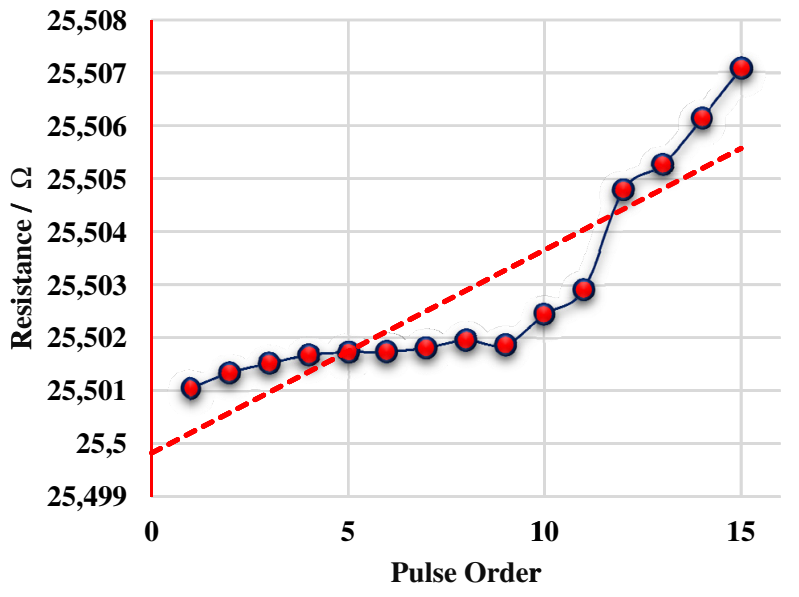

Fig. 5. Overheating increment during TPW realization plateau.

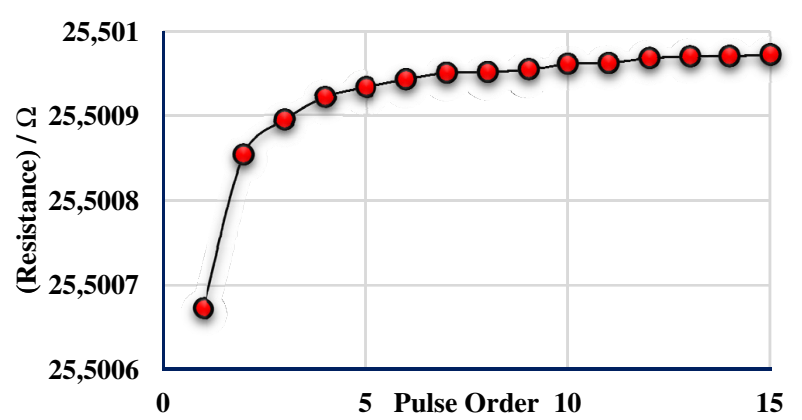

Fig. 6. Plateau values distribution during pulses feeding.

of the system. The static thermal error (compartment heat capacity and thermal resistance) and dynamic thermal error (relaxation and decay time) have been taken into account. The heat capacity $C_{\mathrm{C}}$ of the compartment, the thermal resistance $R_{\mathrm{S}}$ between the compartment,

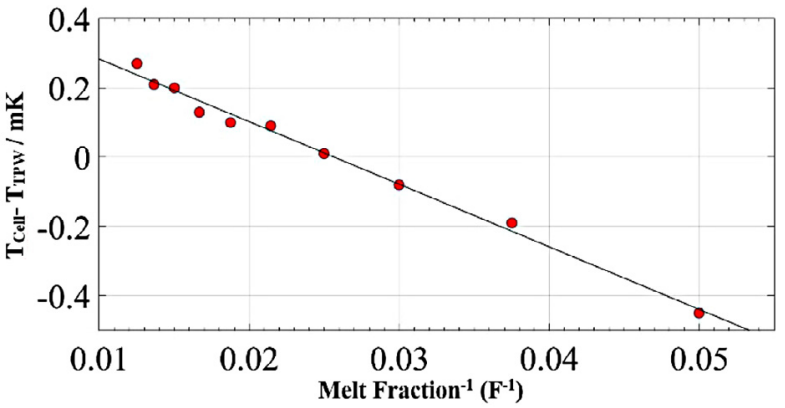

Fig. 7. The extrapolation of a fit to $100 \%$ of $F(20 \% \leqslant F \leqslant$ $80 \%)$.

the adiabatic shield, and the recovery time after each pulse at temperatures close to the TPW.

\subsection{Heat capacity}

The heat capacity of the compartement has a static thermal behaviour while its values did not change by varing the optimum conditions, which was determined by measuring the amount of heat energy required to increase the temperature of the compartment by a known amount $[8,9]$. The energy supplied to the cell was determined from the electrical power applied to the heater around the cell:

$$
C c=\frac{Q}{\Delta T}=\frac{P . t}{\Delta T}
$$

where, $C c(\mathrm{~J} \cdot \mathrm{K}-1), Q(\mathrm{~J}), P(\mathrm{~W})$ and $\Delta T$ are the heat capacity, the amount of thermal heat, the electrical power and the change in temperature after supplying known amount of energy, respectively. Under steady state and stable conditions, an electrical current of $38 \mathrm{~mA}$ was supplied to the $46-\Omega$ heater for 20 min which caused a temperature raise of the compartment by $300 \mathrm{mK}$. The current 
passed through two wires and the other two wires were used to monitor the voltage. From these measurements, the derived heat capacity was $C c=256.696 \pm 0.008 \mathrm{~J} \mathrm{~K}^{-1}$.

\subsection{Thermal resistance}

The thermal resistance between the compartement and the shield has a static thermal behaviour, which was determined as in references $[4,8,9]$ by monitoring both the temperature drifts of the cell and the shield after a sudden change in the temperature of the shield.

$$
R s=\frac{(T s-T c)}{(C c(d T c / d t))}
$$

where, $R_{\mathrm{S}}\left(\mathrm{K} \cdot \mathrm{W}^{-1}\right), T_{\mathrm{S}}(\mathrm{K}), T_{\mathrm{C}}(\mathrm{K})$ and $d T_{\mathrm{C}} / d t\left(\mathrm{~K} \mathrm{~s}^{-1}\right)$ are the thermal resistance, the temperature of the shield, the temperature of the cell and the temperature drift of the cell respectively. Under steady state and stable condition, the regulation set point of the copper shield has been changed by $32 \mathrm{mK}$. The compartment started to follow the rapid change of the shield temperature after several minutes. The temperature drift of the compartment is calculated by change of temperature with respect to the required time for the cell to follow up and stabilize with the new temperature of the shield. The derived thermal resistance was $R_{\mathrm{S}}=6173.516 \pm 0.008 \mathrm{~K} \mathrm{~W}^{-1}$.

\subsection{Recovery time}

The dynamic behaviour of the cell determines the thermal recovery after each heat pulse during the melting fraction plateau $[4,8,9]$. Considering that $\Delta T$ is the temperature difference between the maximum temperature after a heat pulse $T_{\text {Pulse }}$ and the plateau temperature $T_{\text {Plateau }}$

$$
\Delta T=T_{\text {pulse }}-T_{\text {plateau }}
$$

The decay time has been determined as the time interval during which the temperature decreases from $T_{\text {pulse }}$ to $T_{\text {plateau }}$. The TPW cell showed an average decay time close to $7.48 \pm 0.016 \mathrm{~min}$, Table 1 .

\section{Uncertainty Budget}

The uncertainty budget for measurements is shown in Table 2. The estimated uncertainty due to reproducibility was calculated from the scattering of the liquidus point extrapolations in different runs as described above. For the estimation concerning the electrical measurements due to bridge accuracy, standard resistor, multimeters and current sources, it was calculated using the manufacturer's specifications. For the liquidus point estimation, it was calculated from the fitting residuals of the extrapolation of melting fraction $(1 / F)$ for the different runs [10]. The estimated uncertainty due to the heat flux was evaluated from the sensitivity of the plateau temperature to
Table 2. Standard combined uncertainty of TPW realization in the new adiabatic calorimeter.

\begin{tabular}{cc}
\hline Uncertainty components & TPW Cell $/ \mathrm{mK}$ \\
\hline Plateau Reproducibilit [10] & 0.1 \\
Electrical measurement & 0.076 \\
Liquidus point & 0.12 \\
Heat flux & 0.001 \\
Impurities & 0.05 \\
Self-heating & 0.069 \\
Combined uncertainty & 0.19 \\
Expanded uncertainty & 0.37 \\
\hline
\end{tabular}

changes in the control set point of the copper can near the cell [10]. The estimated uncertainty for impurities is calculated through the linear fitting of the relationship between the melted fraction $(1 / F)$ and $\Delta T$ that extrapolated to estimate the liquidus point, which shows slop rate regression of the concentration of impurities (uniform distribution) $[10,11]$. Estimation regarding the self-heating was obtained through performing measurements at $1 \mathrm{~mA}$ and $\sqrt{2} \mathrm{~mA}$. Data were then extrapolated to $0 \mathrm{~mA}[10]$. The data were compared to the scattering values of the thermometer resistance at zero current estimated from $R_{\mathrm{O}}=R_{1}-I_{1}^{2}\left[\frac{R_{2}-R_{1}}{I_{2}^{2}-I_{1}^{2}}\right]$, at $1 \mathrm{~mA}$ and $\sqrt{2} \mathrm{~mA}$.

\section{Conclusion}

Performance and metrological characterization of a new adiabatic calorimeter for the water triple points developed at NIS was discussed. It was noted from the results that there is a slight difference between the metallic-sealed cell and the large cell of $0.1 \mathrm{mK}$ instead of $0.7 \mathrm{mK}$ [2]. This improved difference is due to the improved thermal resistance between the cell and its surroundings (as one part) and could be, as well, due to redistribution of the impurities (other part). In addition, there is an improvement of the uncertainty values of $\pm 0.37 \mathrm{mK}$ compared to those reported from the previous calorimeter of $\pm 0.49 \mathrm{mK}$ [3], where the parasitic heat flux effect was handled by increasing the thermal resistance between the compartment and the vacuum can. The calculated value of impurities concentration was carried out similarly to the method reported in reference [10], it is about of $1.43 \times 10^{-5}$ mole.mole ${ }^{-1}$ which causes a deviation of about $147 \mu \mathrm{K}$. Concerning the intermittent heat technique, more work is still needed in order to assess other thermal parameters such as the realization after freezing the liquid water by two different techniques, the quenching (fast freezing) and smoothing (slow freezing starts at $90 \%$ of melted ice).

\section{References}

1. H. Preston-Thomas, The International Temperature Scale of 1990 (ITS-90), Metrologia 27, 3-10 (1990)

2. A. El Matarawy, M.G. Ahmed, New temperature reference cells for calibrating Capsule Standard Platinum Resistance Thermometers CSPRTs under adiabatic conditions, Int. J. Metrol. Qual. Eng. 5, 401 (2014) 
3. M.G. Ahmed, A. El Matarawy, H.M. Abo Dorra Performance of water and mercury triple points reference cells under adiabatic conditions, J. Phys. Sci. Appl. 1 (2015)

4. F. Sparasci, L. Pitre, Y. Hermier, Realization of the triple point of water in metallic sealed cells at the LNEINM/CNAM: A progress report, Int. J Thermophys. 29, 825-835 (2008)

5. Y. Hermier, L. Pitre, C. Geneville, A. Vergé, G. Bonnier, D.I. Head, B. Fellmuth, L. Wolber, A. Szmyrka-Grzebyk, L. Lipinski, M.J. de Groot, A. Peruzzi, A New generation of multicells for cryogenic fixed points at BNM/INM, AIP Conf. Proc. 684, 179 (2003)

6. M.G. Ahmed, Y. Hermier Degree of equivalence of realizations of the triple points of argon and oxygen between NIS and LNE-Cnam, Int. J. Thermophys. 35, 596-603 (2014)

7. M.G. Ahmed, A.A. El Matarawy, An adiabatic calorimeter for calibrating capsule type thermometers (CSPRTs) in the range $54 \mathrm{~K}$ to $273 \mathrm{~K}$, Int. J. Metrol. Qual. Eng. 3, 85-87 (2012)
8. F. Sparasci, L. Pitre, G. Rouillé, J.-P. Thermeau, D. Truong, F. Galet, and Y. Hermier, An adiabatic calorimeter for the realization of the ITS-90 in the cryogenic range at the LNE-CNAM, Int. J. Thermophys. 32, 201-214 (2011)

9. C. Cappella, F. Sparasci, L. Pitre, B. Buée, A. El Matarawy, Improvements in the realization of the triple point of water in metallic sealed cells at LNE-Cnam, Int. J. Metrol. Qual. Eng. 6, 405 (2015)

10. D.R.White, et al., Uncertainties in the Realization of the SPRT subranges of the ITS-90, Document CCT/0819/rev, http://www.bipm.org/cc/CCT/Allowed/24/D19_ rev_WG3_Doc_rev_10July2009.pdf

11. B. Fellmuth, K.D. Hill, P. Bloembergen, M. de Groot, Y. Hermier, M. Matveyev, A. Pokhodun, D. Ripple, P.P.M. Steur, Methodologies for the estimation of uncertainties and the correction of fixed-point temperatures attributable to the influence of chemical impurities, Working document of the BIPM Consultative Committee on Thermometry, CCT/2005-08 